\title{
Sub-Å STEM Resolution From 30-300kV
}

Maarten Bischoff ${ }^{1}$, Marcel Niestadt ${ }^{1}$, Veli Altin ${ }^{1}$, Alexander Henstra ${ }^{1}$, Peter Tiemeijer ${ }^{1}$, Bert Freitag ${ }^{1}$, Christian Maunders ${ }^{1}$, Peter Hartel $^{3}$ and Eric Van Cappellen ${ }^{2}$

1. Thermo Fisher Scientific, Acht, The Netherlands.

2. Thermo Fisher Scientific, Hillsboro, USA.

3. Corrected Electron Optical Systems GmbH, Heidelberg, Germany.

Interest in low-kV STEM imaging has been fueled by increasing research on novel materials such as carbon-based nano-materials and other low-dimensional materials. Atomic resolution imaging of these classes of materials is seriously affected by radiation damage and weak signals leading to low contrast in the images. Therefore high contrast, beam-damage-free imaging can often only be achieved at low to very low accelerating voltages. Imaging below the critical knock-on damage threshold is the key to success and for some materials this can be well below $60 \mathrm{kV}$.

To maintain optical performance at lower voltages, higher opening angles of the probe have to be used (diffraction limit). Hence the higher order aberrations of the optics, which increase dramatically with higher opening angles and lower voltages, need to be measured and corrected with high precision. Monochromation of the electron beam has proven to be an effective way to reduce chromatic blurring and the Thermo Fisher monochromator - although not specifically developed for imaging - has demonstrated to be a good fit for the purpose of low-kV imaging. The probe corrector can potentially eliminate, or at least significantly reduce, the resolution-limiting aberrations even at low accelerating voltages but some limitations still remain. The corrector must be able to correct up to the $5^{\text {th }}$ order aberrations over the entire high tension range of the tool $(300-30 \mathrm{kV})$ so that optimum imaging performance is achieved for the high tension selected to best suit the material being investigated. Additionally, for correction, the biggest remaining challenge is measuring the aberrations with sufficient confidence to come to reproducible results during daily operation. We will discuss in this presentation how to achieve optimized imaging performance for a wide high tension range, excellent reproducibility of operation and ultimate precision in atomic imaging. With all aberrations corrected, results like the example shown in figure 1 and figure 2 are routinely achievable on a system with full high tension flexibility. 

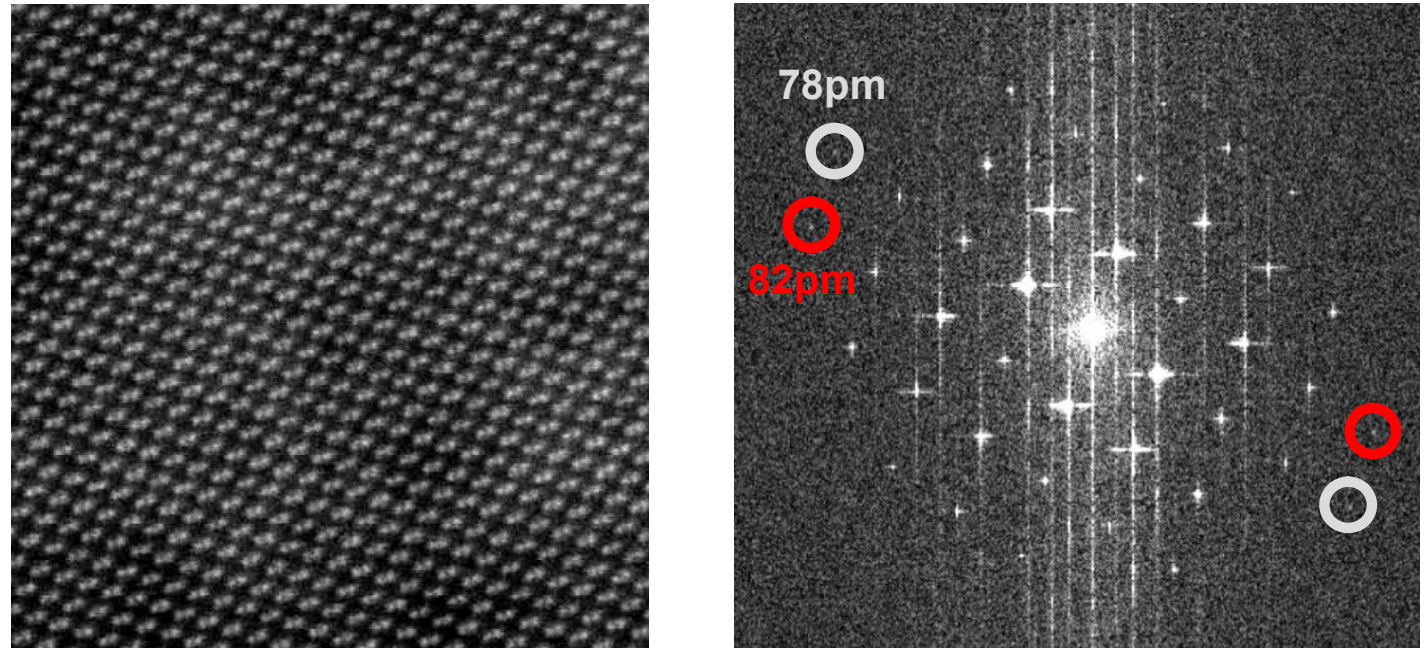

Figure 1. $60 \mathrm{kV}$ ADF of $\mathrm{Si}<110>$ (left ) with FFT of the image (right)

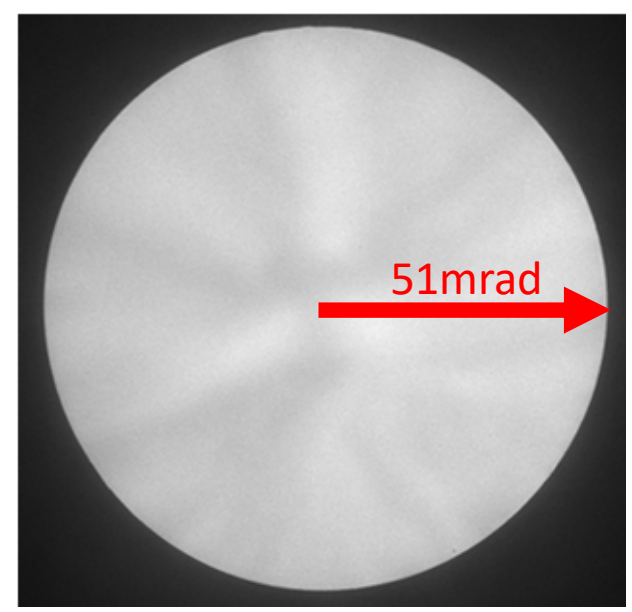

Figure 2. 40kV Ronchigram with $51 \mathrm{mrad}$ convergence semi-angle 